\title{
Correlatos cognitivos y neuropsicológicos de los cinco grandes: una revisión en el área de la neurociencia de la personalidad
}

Jorge Emiro Restrepo*

Doctor en Neuropsicología, Universidad de Salamanca, España. Profesor de tiempo completo, Universidad Cooperativa de Colombia, Medellín, Colombia.

Correo electrónico:

jorge.restrepoc@campusucc.edu.co

Recibido: 20 de febrero del 2015

Aprobado: 22 de mayo del 2015

Cómo citar este artículo: Restrepo,

J. E. (2015). Correlatos cognitivos y

neuropsicológicos de los cinco grandes: una revisión en el área de la neurociencia de la personalidad. Pensando Psicología, 11(18), 107-128. doi: http://dx.doi.org/10.16925/ pe.v11i18.1004

\section{Resumen}

Introducción: la neurociencia de la personalidad es un área de estudio de origen relativamente reciente. Objetivo: estudiar los fundamentos neurobiológicos y neuropsicológicos de la personalidad. Metodología: este es un artículo de revisión narrativa en el cual se presentan y discuten algunas, quizás la mayoría, de las investigaciones realizadas en esta área. La estructura del artículo está en función de los cinco grandes factores de la personalidad. Para cada factor (neuroticismo, extraversión, apertura, amabilidad y responsabilidad) se revisa y discute la literatura existente en relación con los correlatos neuropsicológicos en la función ejecutiva, la memoria y la inteligencia. Resultados: por ser un área de estudio reciente y a razón del tipo de revisión llevado a cabo, no es posible realizar una conclusión precisa, es decir, no es posible establecer, en el estado del arte actual, cómo se correlacionan los factores y las facetas de la personalidad con la función ejecutiva, la inteligencia y la memoria. Conclusión: en términos generales, puede afirmarse que existe un perfil neuropsicológico diferente para cada uno de los factores.

Palabras clave: big five, función ejecutiva, memoria, neuropsicología, personalidad. 


\title{
Cognitive and Neuropsychological Correlates of the Big Five: A Review in the Field of Neuroscience of Personality
}

\begin{abstract}
Introduction: Neuroscience of personality is an area of study whose origins are relatively recent. Objective: The study of the neurobiological and neuropsychological fundamentals of personality. Methodology: This is a narrative review article that presents and discusses some, perhaps the majority, of the research in this field. The structure of the article is based on the five main personality factors. For each factor (neuroticism, extroversion, openness, friendliness and responsibility) the existing literature is reviewed and discussed in relation to the neuropsychological correlates in the executive function, memory and intelligence. Results: Because this is a recent field of study and due to the type of review carried out, it was not possible to reach a precise conclusion; in other words, in the current state-of-the-art, it cannot be determined how the factors and facets of personality correlate with the executive function, intelligence and memory. Conclusion: In general terms, it may be affirmed that there is a different neuropsychological profile for each factor.
\end{abstract}

Keywords: big five, executive function, memory, neuropsychology, personality.

\section{Correlatos cognitivos e neuropsicológicos dos cinco grandes fatores: uma revisão na área da neurociência da personalidade}

\section{Resumo}

Introdução: a neurociência da personalidade é uma área de estudo de origem relativamente recente. Objetivos: estudar os fundamentos neurobiológicos e neuropsicológicos da personalidade. Metodologia: este é um artigo de revisão narrativa no qual são apresentadas e discutidas algumas, talvez a maioria, das pesquisas realizadas nessa área. A estrutura do artigo está em função dos cinco grandes fatores da personalidade. Para cada fator (extroversão, socialização, conscienciosidade, neuroticismo e abertura para experiência), revisa-se e discute-se a literatura existente a respeito dos correlatos neuropsicológicos na função executiva, na memória e na inteligência. Resultados: por ser uma área de estudo recente e pelo tipo de revisão realizado, não é possível realizar uma conclusão precisa, isto é, não é possível estabelecer, no caso da arte atual, como se correlacionam os fatores e as facetas da personalidade com a função executiva, a inteligência e a memória. Conclusão: em termos gerais, pode-se afirmar que existe um perfil neuropsicológico diferente para cada um dos fatores.

Palavras-chave: big five, função executiva, memória, neuropsicologia, personalidade. 


\section{Introducción}

La neurociencia de la personalidad es un área de investigación de origen relativamente reciente. Su objetivo central es el estudio de los fundamentos neurobiológicos y neuropsicológicos de la personalidad. La tesis nuclear de esta disciplina sostiene que cada factor de la personalidad tiene un componente cerebral estructural y funcional, el cual puede ser establecido por medio de un análisis que involucre metodología y técnicas neurobiológicas, neuropsicológicas y de neuroimagen. A la fecha, solo se han desarrollado varias decenas de investigaciones que intentan develar los correlatos neurobiológicos de la personalidad. Menos aún son los estudios que se han interesado por los correlatos neuropsicológicos. La investigación en biología de la personalidad es más antigua que la investigación en neuropsicología de la personalidad.

En esta revisión se presentan y discuten algunas, quizás la mayoría, de las investigaciones realizadas en esta área. El artículo pretende servir como marco integrador de una serie de estudios que hasta ahora no han sido revisados ni discutidos en una publicación en idioma español. Se espera no solo lograr que el lector se informe sobre el estado de arte en esta materia, sino que también encuentre en el artículo un cuerpo articulado de conociminentos sobre un tema de estudio tan relevante y que no pierde vigencia: los fundamentos psicobiológicos de la personalidad.

La estructura del artículo está en función de los cinco grandes factores de la personalidad. Para cada factor (neuroticismo, extraversión, apertura, amabilidad y resposabilidad), se revisa y discute la literatura existente en relación con los correlatos neuropsicológicos en la función ejecutiva, la memoria y la inteligencia. También se revisan y discuten, cuando ha sido posible, la relación entre las facetas de cada factor y sus correlatos neuropsicológicos. No ha sido posible lograr un balance en los resultados de la revisión. Existe mucha más literatura en esta área para factores como el neuroticismo y la extraversión. En el neuroticismo, como se verá, se ha llegado a un alto nivel de detalle en el estudio de los correlatos neuropsicológicos de la función ejecutiva. Pero no ocurre lo mismo con los demás factores.

El artículo condensa una revisión de más de 130 referencias bibliográficas existentes en las principales bases de datos en línea (Apa, DialNet, ovid, Psyc Net, PubMed, Redalyc, Scopus, Springer Link, Science Direct y Wiley InterScience). Se revisaron artículos en español y en inglés. La búsqueda y selección se realizó con base en las palabras clave que incluyeran combinaciones de los nombres de cada factor y cada faceta, así como los términos asociados a los procesos cognitivos y neuropsicológicos. Por ejemplo: "neuroticism and executive function", "extraversion and memory", "neuroticism and cognitive flexibility", "sensation seeking and working memory", etc. No se limitó la búsqueda a un periodo de tiempo específico.

\section{Neuroticismo}

\section{Función ejecutiva}

Williams, Suchy y Kraybill (2010) evaluaron la función ejecutiva en un grupo de adultos mayores. Se interesaron por analizar la flexibilidad cognitiva, la iniciación, la inhibición y la selección de respuestas, la memoria de trabajo, la fluencia generativa y la vigilancia atencional. Para tal fin, utilizaron algunas de las pruebas de la escala de funcionamineto ejecutivo de Delis Kaplan, tales como el trial making test number-letter switching, el design fluency test, el fluency letter y el color-word inhibition switching. El neuroticismo presentó una correlación negativa de $-0,31$ con la medida de funcionamiento ejecutivo. También analizaron las correlaciones entre las facetas y la función ejecutiva. Las correlaciones fueron: ansiedad $(-0,28)$, hostilidad $(-0,44)$, depresión $(-0,33)$, autoconciencia $(-0,16)$, impulsividad $(-0,08)$ y vulnerabilidad $(-0,19)$.

Schretlen, Van der Hulst, Pearlson y Gordon (2010) desarrollaron una investigación, la cual titularon como un estudio neuropsicológico de la personalidad, a fin de examinar las relaciones entre el rasgo de neuroticismo, la inteligencia, la fluencia y el funcionamiento ejecutivo. La correlación del rasgo con el funcionamiento ejecutivo fue de $-0,21$, con la fluencia fue de $-0,13$, con la inteligencia cristalizada fue de $-0,20$, y con la inteligencia fluida la correlación fue de -0,23.

DeYoung, Peterson y Higgins (2005) evaluaron el funcionamiento prefrontal (dorsomedial y dorsolateral) mediante la aplicación de una serie de tareas cognitivas, entre las cuales incluyeron la tarea de la fluencia de palabras, la tarea de la aleatorización de letras, la tarea de la asociación condicional espacial y no espacial, la tarea go/no go y la tarea de la recencia del juicio. Con el fin de obtener la medida del funcionamiento ejecutivo prefrontal, calcularon el promedio de las puntuaciones obtenidas en cada una de las 
tareas. Luego correlacionaron este promedio con las medidas del neuroticismo pero no encontraron ningún tipo de asociación $(0,05)$.

\section{Planificación cognitiva}

Boyle et al. (2010) realizaron un estudio con una muestra de personas mayores de 65 años con el propósito de determinar las relaciones entre el neuroticismo, la depresión y el funcionamiento cognitivo. Mediante una evaluación neuropsicológica que incluyó el Mini mental state examination (MMSE), la escala de demencia de Mattis y el test de apertura de caminos (А у B), la investigación logró establecer que los altos puntajes del rasgo estaban asociados con peores desempeños en el MMSE, pero no se asociaban con el desempeño en la tarea de habilidad de planificación cognitiva. Al analizar la posible mediación de la depresión entre el neuroticismo y el funcionamiento cognitivo, los autores no encontraron ningún efecto moderador de la variable emocional. Los resultados de esta investigación coinciden con los reportados previamente por Jorm et al. (1993) en un estudio similar.

De acuerdo con los resultados del estudio de Portella, Goodwin, Flint, Cowen y Harmer (2004), quienes evaluaron el funcionamiento cognitivo y neuropsicológico en un grupo de personas sanas con altos y bajos niveles de neuroticismo, pero sin depresión ni ansiedad, los altos puntajes del rasgo se correlacionaban con peores desempeños en las tareas cognitivas y neuropsicológicas. En particular, encontraron que estas personas obtenían puntuaciones más bajas en la tarea de procesamiento de información visual rápida, como una medida de atención sostenida, y en la tarea de la Torre de Londres, como una medida de la habilidad de planificación cognitiva.

\section{Flexibilidad cognitiva y rumiación}

En la línea de estudios sobre el análisis de la flexibilidad cognitiva y el neuroticismo se ha sugerido, con un adecuado respaldo empírico, que las personas con altos niveles en el rasgo tienden a tener patrones rígidos e inflexibles de comportamiento y pensamiento (Watson, 1967), aunque se les presenten pruebas incorregibles de que su desempeño está siendo inefectivo a causa de su patrón inflexible (Ingram, 1990). Según sugieren O’ Brien y DeLongis (1996), las personas con un elevado neuroticismo tienden a perseverar en sus pensamientos aun cuando estos son negativos, y no intentan asumir estilos más funcionales y adaptativos, como, por ejemplo, la resolución de problemas.

El estilo rumiativo (o rumiación) se define como una tendencia a estar focalizado sobre los síntomas depresivos o sobre sus causas o consecuencias (Nolen-Hoeksema, 2000). Se ha reportado que el estilo rumiativo aumenta la probabilidad de que los contenidos negativos de la memoria estén más fácilmente disponibles (Lyubomirsky, Caldwell y Nolen-Hoeksema, 1998; Teasdale y Green, 2004), aumenta los niveles de pesimismo (Lyubomirsky, Tucker, Caldwell y Berg, 1999), y puede producir alteraciones en la resolución de problemas y en la toma de decisiones (Lyubomirsky y Nolen-Hoeksema, 1995; Watkins y Moulds, 2005).

Las anteriores características del estilo rumiativo también están asociadas con el neuroticismo, por lo cual sería de esperar que este tuviera algún tipo de relación con aquel. Ya se han reportado este tipo de asociaciones entre el neuroticismo y el estilo rumiativo en población normal (Muris, Roelofs, Rassin, Franken y Mayer, 2005; Wupperman y Neumann, 2006) y población clínica (Bagby y Parker, 2001). Hervas y Vazquez (2011) también reportaron una correlación significativa entre el neuroticismo y el estilo rumiativo. De hecho, Roberts, Gilboa y Gotlib (1998) han sugerido que la rumiación es una importante manifestación cognitiva del neuroticismo.

La rumiación cognitiva se refiere a la persistencia rígida sobre un pensamiento o emoción. Es un estilo inflexible de pensamiento que está relacionado con una disfunción ejecutiva. Según Davis y NolenHoeksema (2000), la rumiación es una manifestación de un patrón más general de perseveración o inflexibilidad cognitiva, lo cual sugiere que las personas que pierden la habilidad para pensar de manera flexible pueden aumentar su tendencia rumiativa. En la rumiación se pierde la capacidad ejecutiva de contener el flujo de pensamiento o de modificar su curso.

\section{Inhibición de respuestas}

La inhibición del comportamiento se ha hallado relativamente asociada con el neuroticismo. En un estudio desarrollado por Muris et al. (2009) en población infantil no clínica, se encontró que el rasgo presentaba correlaciones con la inhibición de respuestas. De acuerdo con Depue e Iacono (1989), y Gray (1987; 1982), existen dos sistemas centrales para el control del comportamiento: el sistema de inhibición (BIS) y el sistema de activación (BAS). Según Gray (1990), 
existen dos subsistemas cerebrales específicos que controlan y regulan cada uno de los dos tipos de control del comportamiento. En la investigación de Smits y Boeck (2006) sobre la relación entre los cinco grandes factores y los dos sistemas de control del comportamiento, se encontró como el neuroticismo estaba positivamente asociado con el sistema de inhibición.

El efecto Stroop tradicionalmente se ha venido utilizando para estudiar la capacidad de controlar la interferencia automática. Se ha conceptualizado como un componente de la función ejecutiva (Reeve y Schnadler, 2001), ya que evalúa la capacidad de cambio de una estrategia inhibiendo la respuesta habitual y ofreciendo una nueva respuesta ante nuevas exigencias estimulares (García y Muñoz, 2000). El neuroticismo se ha asociado con el efecto Stroop. Helode (1982) encontró que este rasgo se correlaciona positivamente con el efecto de interferencia Stroop. Un estudio similar desarrollado por Alperson (1968) no había encontrado asociaciones significativas.

\section{Procesos ejecutivos de la atención}

En general, se ha sugerido que las personas con altos niveles de neuroticismo tienden a tener dificultades en el control de los procesos atencionales (Wallace y Newman, 1998). Los procesos ejecutivos de la atención (control, cambio y focalización), en particular, se han relacionado con el neuroticismo. Muris, De Jong y Engelen (2004) analizaron las correlaciones entre el rasgo y los tres procesos ejecutivos de la atención en un amplio grupo de niños normales. Todas las correlaciones entre el neuroticismo y los tipos de atención fueron negativas, lo cual indica que a mayores niveles del rasgo, menores grados de control atencional $(-0,40)$, focalización atencional $(-0,38)$ y cambio atencional $(-0,34)$.

En otra investigación sobre la relación entre el neuroticismo y los procesos ejecutivos de la atención, Bredemeier, Berenbaum, Most y Simons (2011) encontraron que los altos niveles del rasgo estaban positivamente correlacionados con la dificultad para desenganchar la atención, luego de que esta había sido focalizada en una tarea específica. Koster, Raedt, Goeleven, Franck y Crombez (2005) ya habían demostrado la relación que existía entre los estados emocionales depresivos y la dificultad para desenganchar la antención de estímulos de valencia negativa. El estudio de Bredemeier et al. (2011), no obstante, demostró que esta dificultad para desengancharse no se limita exclusivamente a los estímulos negativos, sino que incluye estímulos de otra naturaleza.
Según la teoría del control atencional (TCA) (Eysenck y Derakshan, 2011; Derakshan y Eysenck, 2009), los fallos atencionales en el rasgo de ansiedad se deben a una falta de eficiencia cognitiva en el procesamiento delainformación (Eysenck, Derakshan, Santos y Calvo, 2007; Eysenck, Payne y Derakshan, 2005). De acuerdo con la TCA, los elevados niveles de ansiedad alterarían la capacidad del sistema cognitivo para inhibir la información irrelevante en la ejecución de una tarea (Berggren, Koster y Derakshan, 2012). Según se ha establecido mediante técnicas de imágen cerebral (IRMf), las personas con altos niveles de ansiedad presentan una disminución en la actividad de la corteza prefrontal dorsolateral (Bishop, 2007; 2009), la cual se supone está encargada de inhibir la información irrelevante. Igualmente, la TCA sugiere que los altos niveles de ansiedad interfieren con la adecuada capacidad para modificar la atención flexiblemente (Derakshan, Smyth y Eysenck, 2009).

\section{Monitorización y regulación}

Luu, Collins y Tucker (2000) encontraron que los altos niveles de neuroticismo estaban asociados con un peor desempeño en las tareas de selección de respuestas, como el test Stroop de colores y palabras. Estos resultados los obtuvieron utilizando potenciales evocados por eventos, a fin de registrar el monitoreo de errores en tiempo real por parte de los participantes en el estudio. Con este procedimiento lograron demostrar que la variabilidad en la negatividad relacionada con los errores estaba en función del estado de ánimo y los estilos de personalidad.

Esta incapacidad para monitorizar y regular los propios procesos (cognitivos o emocionales) fue posteriormente analizada experimentalmente por Bono y Vey (2007) con tareas para evaluar la capacidad de regulación emocional. Este proceso de regulación está bajo el control ejecutivo de los lóbulos prefrontales (García-Andrés, Huertas-Martínez, Ardura y Fernández-Alcaraz, 2010). En este estudio se encontró, igualmente, que los altos niveles de neuroticismo correlacionaban positivamente con peores desempeños en las tareas.

\section{Toma de decisiones}

Denburg et al. (2009) analizaron las relaciones entre los estilos de personalidad y el desempeño en la tarea de apuestas de Iowa (Iowa Gambling Task; Damasio, 
1996), a fin de establecer la variabilidad en los procesos de toma de decisiones en un grupo de adultos mayores. De los cinco factores, encontraron que solo el neuroticismo correlacionaba negativamente con el desempeño en la tarea. Denburg et al. (2009) sugieren que la alteración en este proceso ejecutivo puede deberse al impacto lesivo que podría tener el estrés prolongado sobre el funcionamiento cognitivo.

Hilbig (2008) analizó el proceso de toma de decisiones en las personas con altos niveles de neuroticismo mediante la utilización del heurístico de representatividad. Este heurístico forma parte del grupo de los heurísticos de "rápido" y "frugal" que se definen como cajas de herramientas adaptativas para la toma de decisiones (Goldstein y Gigerenzer, 2002). El estudio de Hilbig (2008) demostró que existía una correlación positiva entre el neuroticismo y la adherencia a la utilización del heurístico de representatividad, lo cual significa que cuanto mayor sea el nivel del rasgo, más probabilidad existe de que la persona asuma como válida la inferencia a partir del heurístico.

\section{Velocidad de reacción y la hipótesis del ruido mental}

Los tiempos de reacción ante tareas cognitivas presentan una variabilidad interindividual que representa diferencias en el procesamiento de información y en la rapidez de la ejecución de operaciones cognitivas. Robinson y Tamir (2005) han indicado que estas diferencias interindividuales, las cuales son más evidentes en las personas con altos niveles de neuroticismo, son el resultado del ruido que se genera en el sistema de procesamiento de la información o de la inestabilidad en las operaciones cognitivas básicas. Robinson y Tamir (2005) sugieren que este ruido podría ser debido a la preocupación y la reactividad.

En el análisis empírico de Robinson y Tamir (2005) sobre la relación entre el neuroticismo y los tiempos de reacción, se encontró que efectivamente existía una marcada diferencia en la ejecución de las tareas cronométricas de reacción, en las cuales los individuos con altos niveles en el rasgo tendían a mostrar menos eficiencia. Esta menor eficiencia parece ser causada por una disfunción ejecutiva, ya que el fallo en el procesamiento puede estar asociado con los mecanismos de control y regulación propios de los lóbulos frontales (Robinson y Tamir, 2005). Belojevic, Jakovljevic y Slepcevic (2003) habían llegado a una conclusión similar luego de realizar una revisión sobre la literatura disponible en el área de estudio de las relaciones entre los estilos de personalidad y el ruido mental (mental noise).

Flehmig, Steinborn, Langner y Westhoff (2007) analizaron las relaciones entre el neuroticismo y los fallos cognitivos en situaciones de la vida diaria, con el objetivo de revisar la hipótesis del ruido mental. También analizaron la relación entre el rasgo y los sitemas de inhibición/activación del comportamiento. Para establecer la primera relación, utilizaron el cuestionario de fallos cognitivos (CFQ), el cual evalúa lapsos o descuidos cognitivos (percepción, atención y memoria) en actividades cotidianas tan sencilas como recordar si se ha apagado o encendido el interruptor de la luz, o como recordar el nombre de una persona que recientemente se ha conocido. Altos puntajes en el $\mathrm{CFQ}$ reflejan déficits en la atención sostenida y en la concentración, así como dificultades generales con la memoria.

En el análisis de Flehmig et al. (2007), se encontraron correlaciones positivas entre el neuroticismo y los puntajes globales del CFQ $(0,26)$. En particular, se presentaron correlaciones entre el CFQ y las categorías de pérdida de activación $(0,18)$, fallos en la activación $(0,20)$ y activación inintencionada $(0,40)$. También se presentó una correlación positiva con la escala de inhibición del comportamiento $(0,39)$. Lo que estos resultados reflejan es como los individuos con altos niveles de neuroticismo tienden a tener intrusiones cognitivas irrrelevantes cuando están realizando una tarea, con lo cual se altera el procesamiento de la información que está en curso. Igualmente, estos individuos tienden a presentar más lapsus de memoria relacionados con el contenido relevante para la ejecución de una tarea en curso. Así mismo, los individuos con altos niveles de neuroticismo tienden a cometer un mayor número de fallos atencionales. Según Flehmig et al. (2007), sus resultados confirman parcialmente la hipótesis del ruido mental.

Colom y Quiroga (2009) también se interesaron por evaluar la hipótesis del ruido mental en el neuroticismo. Sin embargo, sus resultados no encontraron evidencia en favor de tal suposición. En este estudio se evaluó la velocidad de procesamiento mental mediante tres tareas que valoraban la velocidad verbal, numérica y espacial. En cada una de las tareas de velocidad se evaluó la exactitud, el tiempo de reacción y las desviaciones en los tiempos de reacción. Según la suposición teórica de Colom y Quiroga (2009), el componente de la velocidad del procesamiento en las tareas de velocidad se ha vinculado con la periodicidad en el potencial excitatorio de las neuronas. Además, 
las variaciones en las oscilaciones en los periodos de excitación de las neuronas pueden generar un ruido que interfiere con la velocidad de procesamiento cognitivo (Jensen, 1992; 2007). Por tal razón, al evaluar las velocidades de procesamiento se está obteniendo una medida del ruido mental. Los resultados del estudio de Colom y Quiroga (2009) no lograron ofrecer evidencia en favor de un mayor ruido mental en los individuos con altos niveles de neuroticismo.

\section{Memoria}

Gabrys, Schumph y Utendale (1987) reportaron que los procesos de retención y de codificación de memoria eran mejores a corto plazo cuando los individuos tenían bajos niveles de neuroticismo. En este estudio, se les solicitó a los participantes que leyeran una historia que tenía 21 detalles episódicos, los cuales debían ser evocados inmediatamente después de que la persona terminara de leer el texto. El número de detalles evocados estuvo asociado negativamente con el nivel de neuroticismo. Así, cuanto menor era el nivel del rasgo en el individuo, mayor era el número de detalles recordados.

Merema, Speelman, Foster y Kaczmarek (2013) estudiaron las relaciones entre el neuroticismo y la memoria verbal y visual en una muestra de 177 adultos mayores con edades entre los 66 y los 90 años. El valor de la correlación entre el neuroticismo y la memoria visual fue de $-0,094$, mientras que el valor de la correlación entre el factor y la memoria verbal fue de $-0,117$. En esta investigación también se analizó la relación entre el nivel de neuroticismo y el olvido autopercibido o los problemas de memoria autoreportados. La correlación para estas dos variables fue estadísticamente significativa $(\mathrm{p}<0,001)$ y tuvo un valor de $-0,375$.

El neuroticismo se ha asociado con ciertas tendencias (bias) o sesgos (skews) de memoria en los procesos de evocación. En particular, se ha encontrado que los contenidos de memoria asociados a aspectos negativos se recuperan con mayor frecuencia y facilidad en los individuos con altos puntajes en el rasgo. Feldman Barrett (1997) encontró que las personas con altos puntajes en el rasgo sobreestimaban la intensidad promedio de los estados emocionales negativos previamente registrados. Safer y Keuler (2002) le solicitaron a un grupo de personas con altos puntajes en el rasgo - las cuales se encontraban en las últimas sesiones de un proceso psicoterapéutico-recordar su malestar emocional justo antes de comenzar el proceso. Encontraron una tendencia a la sobreestimación de la intensidad del malestar.

Safer, Levine y Drapalski (2002) desarrollaron una investigación con el fin de determinar la relación entre la memoria y los estilos de personalidad, y encontraron una serie de diferencias individuales en la distorsión de la memoria. Luego de realizar el análisis estadístico de regresión múltiple, reportaron que los individuos con altos puntajes en neuroticismo (estudiantes universitarios de licenciatura) tendían a sentirse más ansiosos durante una evaluación escrita y mostraban una tendencia a la sobreestimación de las sensaciones negativas cuando posteriormente se les pedía que las recordaran.

Studer-Luethi, Jaeggi, Buschkuehl y Perrig (2012) analizaron la influencia del rasgo de neuroticismo sobre la capacidad de entrenamiento en tareas de memoria de trabajo en un grupo de 112 estudiantes universitarios de pregrado. El diseño de la investigación se basó en el entrenamiento en tareas sencillas o tareas dobles de n-back por un periodo de cuatro semanas. Antes de iniciar el entrenamiento, se evaluaron las correlaciones entre el neuroticismo y los desempeños en las tareas de memoria de trabajo. La correlación entre el neuroticismo y la tarea de n-back sencilla fue de - 0,07 , y entre el neuroticismo y la tarea de n-back doble fue de -0,01. La investigación estableció que el entrenamiento en la tarea de n-back doble fue más efectivo para los participantes con bajo neuroticismo y que el entrenamiento en la tarea de n-back sencillo fue más efectivo para los participantes con alto neuroticismo.

\section{Inteligencia}

Bartels et al. (2012) desarrollaron un estudio con 2489 gemelos holandeses con el propósito de establecer las relaciones entre la genética, la inteligencia y la personalidad. Utilizando la escala de inteligencia de Wechsler para niños y para adultos, evaluaron el cociente intelectual (CI) verbal, el CI manipulativo y el CI total. La personalidad fue evaluada mediante la versión corta del NEO, el NEO-FFI. Los resultados de los análisis correlacionales mostraron una correlación de -0,10 entre el neuroticismo y el ci total, y una correlación de - 0,12 entre el neuroticismo y el CI verbal. No hubo correlación entre el neuroticismo y el CI manipulativo.

Lounsbury, Welsh, Gibson y Sundstrom (2005) analizaron la relación entre la habilidad cognitiva general, denominada por ellos mismos como 
"inteligencia", y el neuroticismo. Utilizando dos pruebas de aptitud (un test de razonamiento verbal y un test de razonamiento numérico), evaluaron las correlaciones entre estados variables de inteligencia y el Adolescent Personal Style Inventory (APSI), un instrumento de evaluación de los cinco grandes factores de personalidad. El estudio fue realizado con estudiantes de bachillerato de grado medio y grado alto. La edad promedio fue de 11,63 años para los primeros, y de 15,81 para los segundos. La correlación entre el neuroticismo y la medida combinada de inteligencia fue de $-0,15(\mathrm{p}<0,01)$ en los estudiantes de grado medio; $y$ de $-0,13(\mathrm{p}<0.05)$ para los estudiantes de grado alto.

Baker y Bichsel (2006) evaluaron las relaciones entre varias formas de "inteligencia" o habilidades cognitivas, y los cinco grandes factores de personalidad. Siguiendo el modelo de Horn (1985), analizaron: el razonamiento fluido, una habilidad que permite razonar adecuadamente siguiendo procesos inferenciales con el fin de resolver un problema; el conocimiento-comprensión, una habilidad cristalizada o la cantidad y calidad del conocimiento; el pensamiento visuoespacial, una habilidad para comprender configuraciones visuoespaciales; el procesamiento auditivo, una habilidad que permite comprender y discriminar diferentes estímulos auditivos; la velocidad de procesamiento, una habilidad para ejecutar tareas cognitivas automáticas con rapidez; la memoria a corto plazo, una habilidad para retener y recuperar información por un corto lapso de tiempo; y la memoria a largo plazo, una habilidad para recuperar información luego de un largo lapso de tiempo. Luego de realizar un análisis de regresión, Baker y Bichsel (2006) no encontraron ninguna relación entre el neuroticismo y los diferentes tipos de habilidades cognitivas.

Para el neuroticismo, los resultados de las relaciones con las diferentes habilidades cognitivas o formas de 'inteligencia', han sido contradictorios. Pearson (1993) reportó una correlación positiva entre el neuroticismo y una medida de la inteligencia cristalizada, o la habilidad cognitiva tipo comprensión-conocimiento, en mujeres mayores diagnosticadas con depresión y ansiedad. Jorm et al. (1993) reportaron correlaciones negativas entre el neuroticismo, el razonamiento fluido y la memoria a corto plazo en hombres; y correlaciones negativas entre el neuroticismo y la velocidad de procesamiento en mujeres. Jelicic et al. (2003), al igual que Baker y Bichsel (2006), no reportaron correlaciones entre ninguna de las medidas de los diferentes tipos de habilidades cogntivas y el neuroticismo. Furnham, Forde y Cotter (1998) encontraron que algunas de las facetas del neuroticismo sí correlacionaban con algunas habilidades cognitivas. Por ejemplo, reportaron que la impulsividad correlacionaba positivamente con el razonamineto fluido.

\section{Extraversión}

\section{Función ejecutiva}

En la investigación de Williams, Suchy y Kraybill (2010; presentada en el apartado "Función ejecutiva" de "Neuroticismo"), la extroversión presentó una correlación de 0,01 con la medida de funcionamiento ejecutivo. También analizaron las correlaciones entre las facetas y la función ejecutiva. Las correlaciones fueron las siguientes: afectividad $(0,32)$, búsqueda de sensaciones $(-0,23)$, asertividad $(-0,1)$, gregarismo $(0,02)$, emociones positivas $(0,21)$, y proactividad $(-0,06)$. El estudio de DeYoung, Peterson y Higgins (2005) reportó una correlación negativa muy baja $(-0,10)$ entre el funcionamiento ejecutivo y la extraversión.

Ramchandran (2011) analizó los correlatos neuropsicológicos en la función ejecutiva del liderazgo y la efectividad. Estas dos funciones han sido ya establecidas como características, o facetas de la extroversión (Campbell, Simpson, Stewart y Manning, 2003). El estudio utilizó la tarea de apuestas de Iowa, la tarea de la Torre de Hanoi, el test de fluencia figurativa de Ruff, el test de fluencia de categorías, el test Stroop, el test de apertura de caminos forma B y la tarea de aplicación de estrategias, entre otros instrumentos para la medición del funcionamineto ejecutivo.

Las correlaciones encontradas entre la extroversión y las diferentes medidas de la función ejecutiva fueron las siguientes (Ramchandran, 2011): - 0,014 para la tarea de la Torre de Hanoi; 0,194 para el test de fluencia figurativa de Ruff; -0,018 para el test de fluencia de categorías; -0,017 para el test Stroop; 0,070 para el test de apertura de caminos forma B; y 0,000 para la tarea de aplicación de estrategias. En términos generales, el estudio no encontró una asociación entre la extroversión y la función ejecutiva. En el estudio de Schretlen, Van der Hulst, Pearlson y Gordon (2010) (presentado en el apartado "Función ejecutiva") la correlación con el funcionamiento ejecutivo fue de 0,08 , con la fluencia fue de 0,16 , con la inteligencia cristalizada fue de 0,12 , y con la inteligencia fluida la correlación fue de 0,18. 


\section{Memoria de trabajo}

Gray y Braver (2002) analizaron la relación entre la dimensión extroversión/introversión y la activación neuronal en la región caudal del cortex cingulado anterior. Esta zona es crítica para el control cognitivo del comportamiento y está asociada con el funcionamiento de la memoria de trabajo cuando esta tiene una alta carga de información desde la cual operar (Botvinick, Braver, Barch, Carter y Cohen, 2001). El estudio se sirvió de una tarea de n-back (una tarea de 3 back) para evaluar la capacidad en la memoria de trabajo de los participantes. El propósito era determinar si el sistema de activación del comportamiento (BAS) se asociaba con un mejor funcionamiento en la memoria de trabajo, así como con una menor activación en la corteza cingulada anterior. De igual forma, si el sistema de inhibición del comportamiento (BIS) se asociaba con un peor funcionamiento en la memoria de trabajo y una mayor activación en la corteza cingulada anterior.

La investigación de Gray y Braver (2002) logró corroborar los hallazgos del estudio de Carver y White (1994) sobre el BIS y el BAS en relación con los estilos de personalidad extrovertidos/introvertidos. En particular, encontraron que los individuos extrovertidos tendían a presentar mayores niveles de activación comportamental, tal y como lo habían demostrado Lieberman y Rosenthal (2001), y un mejor desempeño en la tarea de memoria de trabajo. Este último estudio puso en evidencia que los extrovertidos pueden mantener más de un objetivo relevante en su memoria de trabajo, mientras que los introvertidos solo podían conservar uno.

Lieberman (2000) ya había encontrado que los individuos extrovertidos tenían mejores desempeños en tareas de memoria de trabajo en comparación con los introvertidos. Según él, la diferencia entre el funcionamiento de ambos grupos radica en que los primeros tienen un ejecutivo central (Baddeley, 1986) más activo, en razón a la influencia del funcionamiento de la formación reticular, que presenta una activación diferencial entre los dos grupos, siendo menor para los extrovertidos (Eysenck, 1967).

\section{Flexibilidad cognitiva}

Campbell, Davalos, McCabe y Troup (2011) evaluaron la flexibilidad cognitiva en un grupo de individuos extrovertidos, introvertidos y ambivertidos por medio del test de clasificación de tarjetas de
Winsconsin (พСsт). Los valores de las medias para los tres grupos sugieren que los individuos extrovertidos son superados por los otros dos grupos en la ejecución del test. El valor de las medias para los errores y las respuestas perseverativas es mayor en los individuos extrovertidos en comparación con los introvertidos y los ambivertidos.

Dreisbach y Goschke (2004) encontraron que la afectividad positiva y la extroversión aumentaban la flexibilidad cognitiva y reducían las respuestas perseverativas pero lo hacían aumentando a su vez la distractibilidad. Los autores sugieren que estos efectos de la afectividad positiva y la extroversión sobre la flexibilidad cognitiva se dan en razón a un aumento en los niveles de dopamina en ciertas regiones frontales del cerebro. Anteriormente se habían hallado relaciones similares entre la extroversión, la afectividad positiva y el aumento de la flexibilidad mental (Isen y Daubman, 1984; Isen, Niedenthal y Cantor, 1992).

El afecto positivo, una de las facetas de la extroversión, favorece la flexibilidad cognitiva y facilita la creatividad en la solución de problemas (Isen, Daubman y Nowicki, 1987; Isen y Means, 1983). En especial, se ha relacionado con la toma de decisiones y la organización cognitiva. Según lo refiere Isen (1984), cuando las personas reportan sentirse bien tienden a tomar decisiones de forma más creativa y efectiva. De manera que las personas con afectividad positiva tienden a resolver el problema de la vela (candle task) (Duncker, 1945) con más facilidad, ya que su flexibilidad cognitiva les permite superar el efecto de fijeza funcional ( functional fixedness) que caracteriza los estilos inflexibles de pensamiento.

\section{Inhibición de respuestas}

De acuerdo con la teoría de la extraversión desarrollada por Brebner (1980; 1985), existe un hipotético sistema de procesamiento central que puede estar excitado o inhibido. Cada uno de estos dos estados estaría en función de la demanda para el análisis de un estímulo o de la organización de la respuesta. Tal y como se sugiere, los individuos introvertidos obtienen excitación del análisis de los estímulos, mientras que los extrovertidos producen la excitación a partir de los procesos involucrados en la organización de la respuesta. Stahl y Rammsayer (2007) consideran que, guardando consistencia con estas hipótesis, los individuos extrovertidos deberían tener una respuesta motora más rápida que los introvertidos. 
En su investigación, Stahl y Rammsayer (2007) analizaron la capacidad de inhibición de respuestas motoras con un paradigma de señal de parada (stop-signal) en un grupo de individuos extrovertidos. Con este paradigma, los participantes se involucran en una tarea de elección-reacción, y de vez en cuando y de manera impredecible, se presenta una señal de parada que les obliga a inhibir su respuesta. Este paradigma se ha utilizado para investigar los llamados "modelos de carrera de caballos" (horse race models; Stahl y Gibbons, 2007). Este modelo describe una "carrera" entre dos sistemas independientes pero concurrentes: uno que controla la respuesta motora (go system) y otro que controla la respuesta en curso (stop system). Ambos sistemas son activados por las señales de inicio y parada.

Stahl y Rammsayer (2007) pretendieron, entonces, demostrar que los altos niveles de extraversión estaban asociados con un nivel aumentado de excitación motora inducida por una tarea. Efectivamente, los resultados de su estudio demostraron que los individuos con altos niveles de extraversión presentaban un menor control inhibitorio (una correlación de - 0,35 con el tiempo de reacción), como lo reflejaron las puntuaciones en los tiempos de reacción para la señal de parada cuando se comparan con los de los individuos introvertidos. Igualmente, la extroversión correlacionó positivamente con la impulsividad disfuncional.

Campbell, Davalos, McCabe y Troup (2011) evaluaron la inhibición de respuestas en un grupo de individuos extrovertidos, introvertidos y ambivertidos mediante la aplicación de la Torre de Hanoi, la tarea de go/no go afectiva y la tarea Anti-cue (Salthouse, Atkinson y Berish, 2003). Los individuos extrovertidos cometieron más errores en las dos últimas tareas en comparación con los otros dos grupos. Solo demostraron tener un mejor rendimiento en la prueba de la Torre de Hanoi, que no es precisamente un prueba para evaluar inhibición, sino para la evaluación de la planificación cognitiva y el aprendizaje (Goel y Grafman, 1995).

\section{Memoria}

Howarth y Eysenck (1968) elaboraron un diseño experimental con dos grupos de individuos introvertidos y extrovertidos a fin de analizar las diferencias individuales en los procesos de retención y evocación de memoria a corto y largo plazo. El estudio utilizó siete pares de asociaciones con intervalos de retención de $0,1,5$ y 30 minutos, y 24 horas. Los resultados mostraron que los individuos extrovertidos tenían mejores desempeños en los intervalos a corto plazo, pero eran inferiores en comparación con los introvertidos cuando la evaluación se realizaba con intervalos a largo plazo. Según Eysenck (1967), el bajo nivel de arousal de los extrovertidos no facilita la codificación a largo plazo, pero tiene mucha incidencia en el proceso a corto plazo.

Gabrys, Schumph y Utendale (1987) también encontraron que los procesos de retención y de codificación de memoria eran mejores a corto plazo cuando los individuos tenían altos niveles de extroversión. En este estudio, se les solicitó a los participantes que leyeran una historia que tenía 21 detalles episódicos que debían ser evocados inmediatamente después de quela persona terminara de leer el texto. El número de detalles evocados estuvo asociado positivamente con el nivel de extraversión. Así, cuanto mayor era el nivel del rasgo en el individuo, mayor era el número de detalles recordados.

Tal y como reconocen Sanford y Fisk (2009), las diferencias individuales entre los extrovertidos y los introvertidos están relacionadas con varios aspectos de los procesos de la memoria, como la evocación (Bermúdez, Pérez y Padilla, 1988) y el reconocimiento. Gupta y Kumar (1990) encontraron que la codificación eleborada aumentaba el desempeño en el proceso de evocación de memoria en los individuos extrovertidos, mientras que este efecto no se presentó en los introvertidos. Los resultados del estudio de Schwartz (1979) mostraron que los individuos extrovertidos accedían de forma más eficiente a la memoria semántica cuando realizaban juicios sobre categorías taxonómicas.

Harley y Matthews (1992) analizaron el efecto del rasgo sobre el priming. En general, los individuos extrovertidos mostraron más priming semántico en comparación con los introvertidos. Los extrovertidos lograron reconocer o clasificar con mayor rapidez una palabra objetivo cuando esta ya había sido cebada (primed) sistemáticamente con una palabra relacionada. Según Harley y Matthews (1992), estos efectos eran sensibles al nivel de arousal y a la hora del día. El mayor efecto de priming se producía cuando los individuos reportaban mayores niveles de arousal y en las horas de la mañana.

Frost, Sparrow y Barry (2006) analizaron la relación entre la dimensión introversión/extraversión y la generación de falsas memorias. A un grupo de 
personas se les pidio que vieran un video en el cual ellos actuaban como testigos. Luego de revisar el video, se les interrogaba sobre algunos de los aspectos que observaron a lo largo de las escenas. A los participantes se les pidió que respondieran a todas las preguntas, aun si no tenían claridad sobre la respuesta.

Todas las preguntas, a excepción de las cuatro finales, tenían relación con el video e indagaban sobre asuntos que efectivamente habían sucedido. Las cuatro últimas preguntas interrogaban sobre aspectos que no habían sucedido en el video, pero que debían ser contestadas por los participantes, de manera que los obligaba a confabular. Cuando los participantes contestaban a las primeras preguntas (las verdaderas) recibían un feedback neutral (e.g. "Ok"), pero cuando respondieron a las preguntas en las que debían confabular recibían un feedback confirmatorio (e.g. "Es correcto. Esa es la respuesta correcta").

Luego de una semana, los participantes regresaban para contestar nuevamente una serie de preguntas sobre el video. En esta nueva sesión se les informaba que el entrevistador de la primera sesión había cometido algunos errores y les había preguntado sobre aspectos que nunca habían ocurrido realmente en el video. Se les pedía que solo respondieran con base en lo que recordaban del video. Los resultados de las correlaciones entre las puntuaciones y los estilos de personalidad demostraron como los individuos extrovertidos eran más suceptibles a los falsos reconocimientos de eventos asociados con feedbacks confirmatorios, en comparación con los introvertidos (Frost, Sparrow y Barry, 2006). Los autores sugieren que esta diferencia puede explicarse debido a que los individuos extrovertidos tienden a estar más atentos a las claves sociales (social cues).

\section{Atención}

De acuerdo con la teoría de Eysenck (1967) sobre los diferentes niveles de arousal en los individuos introvertidos y extrovertidos, sería de esperar que los primeros tuvieran un mejor desempeño en tareas de atención sostenida y en condiciones de monotonía ambiental, ya que su nivel de activación tónica les permitiría mantener la atención con relativa independencia de las propiedades del estímulo. La investigación de Matthews, Davies y Lees (1990) ofreció evidencia a favor de esta posición teórica. Los resultados demostraron una relación lineal entre el arousal y el desempeño en las tareas de atención sostenida.
Además, lograron establecer que las demandas de la tarea influían sobre el efecto de la extraversión.

Los resultados del metaanálisis de Koelega (1992) sobre la extraversión/introversión y la vigilancia no fueron completamente consistentes con estas suposiciones teóricas. Los resultados primarios del metaanálisis sugirieron que el desempeño de los introvertidos en las tareas de atención sostenida era mejor que el de los extrovertidos. Sin embargo, un análisis posterior, con una muestra más amplia que la primera y con un tamaño del efecto mayor, demostró que los individuos introvertidos tenían mejor desempeño en todas las tareas relacionadas con detección, pero no tenían un buen desempeño en las tareas de mantenimiento de la atención a lo largo del tiempo. Buela Casal, Caballo, García Cueto y Flores Cubos (1990) no encontraron ninguna diferencia estadísticamente significativa entre los desempeños en tareas de atención entre los introvertidos y los extrovertidos.

\section{Inteligencia}

En el estudio de Bartels et al. (2012) los resultados de los análisis correlacionales mostraron una correlación de -0,03 entre la extraversión y el ci manipulativo, y una correlación de - 0,02 entre la extraversión y el cI verbal. No hubo correlación entre la extraversión y el Ci total. En la investigación de Lounsbury, Welsh, Gibson y Sundstrom (2005), presentada en el apartado "Inteligencia" en "Neuroticismo", la correlación entre la extraversión y la medida combinada de inteligencia fue de 0,19 ( $\mathrm{p}<0,01)$ en los estudiantes de grado medio; y de $0,22(\mathrm{p}<0,01)$ para los estudiantes de grado alto. Baker y Bichsel (2006), en el estudio comentado anteriormente, encontraron que la extraversión en personas jóvenes era un predictor de la habilidad tipo conocimiento-comprensión $(\beta=-0,19)$, y de la habilidad tipo velocidad de procesamiento $(\beta=-0,20)$.

Roberts (2002) reportó que las personas introvertidas tenían mejores desempeños en las tareas que medían la habilidad tipo conocimiento-comprensión cuando eran comparados con personas con niveles intermedios o altos de extraversión. Stough et al. (1996) habían reportado previamente que las personas con niveles intermedios de extraversión tenían mejores desempeños en las medidas de razonamiento fluido y conocimiento-comprensión, en comparación con personas con niveles bajos (introversión) o muy altos en el rasgo. 


\section{Apertura a la experiencia}

\section{Función ejecutiva}

DeYoung, Peterson y Higgins (2005), presentado en el apartado "Función ejecutiva" de "Neuroticismo", reportaron una correlación positiva de 0,21 entre el funcionamiento ejecutivo y la apertura a la experiencia. En esta misma investigación se encontraron correlaciones significativas entre la apertura a la experiencia y varias subpruebas de la escala de inteligencia para adultos de Wechsler (WAIS III). Las subpruebas de la escala y las correlaciones con el rasgo fueron las siguientes: vocabulario $(0,33)$, semejanzas $(0,27)$, diseño con bloques $(0,16)$, aritmética $(0,18)$, y dígitos y símbolos $(-0,18)$. También aplicaron la escala avanzada del test de matrices progresivas de Raven y establecieron una correlación de 0,23.

El estudio de DeYoung, Peterson y Higgins (2005) también analizó las correlaciones entre las facetas de la apertura a la experiencia y varias de las medidas de funcionamiento ejecutivo. Solo encontraron correlaciones significativas entre: la apertura a la estética y la atención selectiva y la memoria de trabajo $(0,15)$, evaluada mediante el self-ordered pointing test; la apertura a los sentimientos y la atención selectiva y la memoria de trabajo $(0,15)$, evaluada mediante el letter randomization task; la apertura a las ideas y la memoria de trabajo $(0,20)$, evaluada mediante la tarea de la asociación condicional espacial; la apertura a las ideas y la atención sostenida y la inhibión de respuestas $(0,15)$, evaluada mediante la tarea de go/no go; la apertura a los valores y la atención sostenida y la inhibión de respuestas $(0,17)$, evaluada mediante la tarea de go/no go; la apertura a los sentimientos y el test de fluencia de palabras $(0,16)$; la apertura a las ideas y el test de fluencia de palabras $(0,20)$.

En el estudio de Williams, Suchy y Kraybill (2010), la apertura a la experiencia presentó una correlación positiva de 0,28 con la medida compuesta de funcionamiento ejecutivo. Esta investigación también se interesó por analizar las correlaciones entre las diferentes facetas del rasgo y la medida de la función ejecutiva. Para la apertura a la estética la correlación fue de 0,14; para la apertura a la fantasía de 0,21; para la apertura a los sentimientos de 0,20; para la apertura a las ideas de 0,23 ; para la apertura a la acción de 0,22 ; y para la apertura a los valores la correlación fue de 0,21. En general, las correlaciones entre el funcionamiento ejecutivo y las facetas fueron unas de las más altas reportadas en la investigación.

\section{Inteligencia}

Si se consideran los altos desempeños en tareas ejecutivas que han demostrado las personas con altos niveles del rasgo de apertura a la experiencia, sería acertado considerar que el rasgo podría mostrar una correlación positiva con el desempeño en tareas que evalúan el funcionamiento cognitivo general, como las pruebas de inteligencia (Costa y McCrae, 1992). Así, por ejemplo, McCrae (1993-1994) encontró una correlación de 0,33 entre la apertura (NEO-PI-R) y el IQ (Intellegenz-Quotient) evaluado mediante la escala de inteligencia de Wechsler (waIs). Holland, Dollinger, Holland y MacDonald (1995) reportaron una correlación de 0,42 entre la apertura (NEO-PI-R) y el IQ evaluado mediante la escala de inteligencia de Wechsler revisada (WAIS-R).

Ashton, Lee, Vernon y Jang (2000) se interesaron por analizar las relaciones entre la inteligencia fluida, la inteligencia cristalizada y la apertura a la experiencia. El rasgo mostró las correlaciones más altas y significativas con las medidas de la inteligencia cristalizada, evaluada con pruebas de vocabulario, semejanzas, información, comprensión, organización de dibujos y ensamblaje de cubos. La correlación global entre la inteligencia cristalizada y la apertura a la experiencia fue de 0,37 . La correlación global entre la inteligencia fluida y el rasgo fue de 0,18 . La correlación entre la medida global de la inteligencia y la apertura a la experiencia fue de 0,29. En el estudio de Schretlen, Van der Hulst, Pearlson y Gordon (2010), presentado en el apartado "Función ejecutiva" de "Neuroticismo", la correlación con el funcionamiento ejecutivo fue de 0,16 , con la fluencia fue de 0,24 , con la inteligencia cristalizada fue de 0,44 , y con la inteligencia fluida la correlación fue de 0,32.

Wainwright, Wright, Luciano, Geffen y Martin (2008) examinaron las correlaciones de las facetas de la apertura a la experiencia con una serie de medidas de inteligencia, tiempos de reacción, tiempos de inspección y logro académico en una muestra de más de 700 individuos. Las correlaciones más altas fueron las siguientes: apertura a las ideas y IQ verbal $(0,40)$, apertura a los valores e IQ verbal $(0,26)$, apertura a los sentimienos e IQ verbal $(0,20)$, apertura a las ideas e IQ manipulativo $(0,28)$, apertura a los sentimientos y logro académico $(0,24)$, apertura a las ideas y logro académico $(0,42)$, apertura a los valores y logro académico $(0,32)$. No se encontró ninguna correlación relevante entre las facetas y los tiempos de reacción e inspección. 
El conservadurismo se ha definido como un rasgo opuesto al de apertura a la experiencia (Stankov, 2009). En contraposición a la apertura, este rasgo se caracteriza por una marcada tendencia al respeto por la tradición y un exceso de conformismo. Las personas con bajos puntajes en el rasgo de apertura son convencionales, estructuradas y persistentes en sus juicios. Tienen una mayor necesidad de orden y manifiestan un comportamiento más constreñido. Stankov (2009) realizó un análisis de las relaciones entre los bajos puntajes en el rasgo de apertura a la experiencia (o altos niveles de conservatismo) y los puntajes en la prueba de aptitud escolar (sAT) en un grupo de 733 estudiantes que aspiraban a ingresar a la universidad y encontró una correlación negativa de $-0,35$.

El estudio de Bartels et al. (2012) (presentada en el apartado "Memoria" de "Neuroticismo"), reportó una correlación de 0,32 entre la apertura a la experiencia y el cr total, una correlación de 0,24 entre la apertura a la experiencia y el CI manipulativo, y una correlación de 0,33 entre la apertura y el CI verbal. La investigación de Lounsbury, Welsh, Gibson y Sundstrom (2005), presentada en el apartado "Memoria" de "Neuroticismo", encontró que la correlación entre la apertura a la experiencia y la medida combinada de inteligencia fue de $0,13(\mathrm{p}<0,01)$ en los estudiantes de grado medio; y de 0,25 ( $p<0,01)$ para los estudiantes de grado alto. Baker y Bichsel (2006), presentada en el apartado "Memoria" de "Neuroticismo", encontraron que la apertura a la experiencia en personas jóvenes era un predictor de la habilidad tipo Conocimiento-comprensión $(\beta=0,27)$ y de la habilidad tipo Memoria a largo plazo $(\beta=0,19)$. En adultos mayores encontraron que la apertura a la experiencia era un predictor de la habilidad tipo Pensamiento visuoespacial $(\beta=0,21)$.

\section{Amabilidad}

\section{Función ejecutiva}

En el estudio de Williams, Suchy y Kraybill (2010), la amabilidad presentó una correlación positiva de 0,38 con la medida de funcionamiento ejecutivo. Esta correlación fue la más alta en comparación con los demás factores. También analizaron las correlaciones entre las facetas y la función ejecutiva. La correlación con la modestia fue de - 0,06 . La correlación con la sinceridad fue de 0,09. La correlación con el altruismo fue de 0,31 . La correlación con la confianza fue de
0,44. La correlación con la complacencia fue de 0,29. La correlación con la simpatía fue de 0,33 . DeYoung, Peterson y Higgins (2005) no encontraron ningún tipo de asociación $(-0,08)$ entre el funcionamiento ejecutivo y el factor amabilidad.

Schretlen, Van der Hulst, Pearlson y Gordon (2010), en su estudio neuropsicológico de la personalidad, examinaron las relaciones entre el rasgo de amabilidad, la inteligencia, la fluencia y el funcionamiento ejecutivo. La correlación del rasgo con el funcionamiento ejecutivo fue de 0,09 , con la fluencia fue de 0,15 , con la inteligencia cristalizada fue de 0,25 , y con la inteligencia fluida la correlación fue de 0,20. La empatía y la prosocialidad son facetas de la amabilidad y están fuertemente asociadas con esta (Gray, 2009). Puskar (2011) logró establecer la estrecha relación entre el funcionamiento ejecutivo y la empatía mediante una evaluación extensa y profunda con una serie de pruebas cognitivas y afectivas que valoraban la integridad funcional de los lóbulos prefrontales.

Rameson y Lieberman (2009) ofrecen una excelente revisión sobre los fundamentos cognitivos y neuropsicológicos de la empatía en relación con el funcionamiento ejecutivo. En particular, destacan la importancia de la corteza prefrontal medial (CPFM) como parte fundamental del circuito que subyace al funcionamiento de la teoría de la mente. Por otra lado, ya se conocen las relaciones neurocognitivas entre el funcionamiento ejecutivo y la teoría de la mente (Joseph y Tager-Flusberg, 2004; Rasmussen, Wyper y Talwar, 2009).

\section{Control ejecutivo e inhibición de respuestas}

Williams, Suchy y Rau (2009) sostienen que algunas de las habilidades ejecutivas influencian la expresión de la amabilidad. Tal y como lo refieren Ahadi y Rothbart (1994), la amabilidad está vinculada, durante el desarrollo psicológico, al control esforzado (effortful control) del temperamento. Muris, Bos, Mayer, Verkade, Thewissen y Dell'Avvento (2009) encontraron que la amabilidad estaba negativamente correlacionada con la inhibición, evaluada mediante el cuestionario de inhibición comportamental. Bettencourt, Talley, Benjamin y Valentine (2006) reportaron que la amabilidad se asociaba negativamente con las tendencias agresivas, incluso en condiciones de provocación, lo cual indica un fuerte control y regulación comportamental.

Según Tobin, Graziano, Vanman y Tassinary (2000), la capacidad de inhibir las tendencias antipáticas y displicentes, sobre todo las relacionadas con 
expresiones emocionales que pueden afectar la calidad de las relaciones interpersonales, es fundamental para la configuración y el funcionamiento de la amabilidad. Flory et al. (2006) analizaron la relación entre el rasgo y la impulsividad disposicional, la cual se caracteriza por la búsqueda de novedad, la desinhibición del comportamiento y la no planificación. Como era de esperarse, el rasgo correlacionó negativamente con la impulsividad. Según esto, las personas con altos niveles de amabilidad tienden a tener mayor inhibición y control sobre sus comportamientos.

Tangney, Baumeister y Boone (2004) reportaron una asociación entre la amabilidad y una medida de autocontrol que evaluaba la habilidad para controlar las demandas comportamentales, tales como la necesidad de comer en exceso y la pereza. Jensen-Campbell, Rosseli, Workman, Santisi, Rios y Bojan (2002) ya habían encontrado que el rasgo estaba asociado con un menor efecto de interferencia tipo Stroop, lo que significaba que estas personas tenían mejores habilidades para inhibir conflictos cognitivos.

El control con esfuerzo (effortful control) es un constructo cognitivo primario que ha sido vinculado teóricamente con las regiones de control cognitivo del cerebro. Se ha definido como "la eficiencia de la atención ejecutiva, incluyendo la habilidad para inhibir una respuesta dominante y/o activar una respuesta subdominante, planear y detectar fallos" (Rothbart y Bates, 2006, p. 129). Se caracteriza por la capacidad de enfocar conscientemente la atención a fin de inhibir voluntariamente o iniciar algunos comportamientos, tales como el retraso de la acción.

Eisenberg et al. (1996) sugirieron que la amabilidad debería estar asociada con la auto regulación y el control con esfuerzo en los procesos emocionales normales y clínicos. La investigación de Ode y Robinson (2007) pudo establecer que los niveles de amabilidad eran determinantes para controlar y disminuir algunas quejas somáticas asociadas al neuroticismo y causadas, en parte, por el escaso control y regulación emocional que caracteriza a este rasgo. Según su suposición, la cual fue confirmada luego de los análisis estadísticos, ciertos grados de amabilidad podrían mitigar el efecto del neuroticismo sobre las emociones.

\section{Atención}

En la neurociencia de la visión se ha estudiado un proceso cognitivo que se conoce como desenganche atencional. Según Posner y Petersen (1990), el desenganche se refiere a la interrupción en el procesamiento de un estímulo para cambiar de atención hacia un estímulo diferente. Wilkowski, Robinson y Meier (2006) lograron determinar que las personas con bajos niveles de amabilidad tenían mayores dificultades para desengancharse de estímulos antisociales, mientras que las personas con altos niveles del rasgo tenían dificultades para desengancharse de estímulos prosociales. Según ellos, el nivel del rasgo es un predictor del estilo cognitivo para el procesamiento de la información social. De tal manera que la existencia del rasgo puede introducir sesgos en función de la naturaleza del estímulo.

\section{Inteligencia}

Los resultados de los análisis correlacionales, en el estudio de Bartels et al. (2012; presentado en el apartado "Memoria" de "Neuroticismo"), mostraron una correlación de 0,21 entre la amabilidad y el CI total, una correlación de 0,18 entre la amabilidad y el CI manipulativo, y una correlación de 0,19 entre la amabilidad y el ci verbal. Por otra parte, la correlación entre la apertura a la experiencia y la medida combinada de inteligencia fue de $0,11(\mathrm{p}<0,05)$ en los estudiantes de grado medio; y de $0,14(\mathrm{p}<0,01)$ para los estudiantes de grado alto, según los análisis de Lounsbury, Welsh, Gibson y Sundstrom (2005). Luego de realizar un análisis de regresión, Baker y Bichsel (2006) encontraron que la apertura a la experiencia en personas jóvenes era un predictor de la habilidad tipo Conocimiento-comprensión $(\beta=0,27)$, así como de la habilidad tipo Memoria a largo plazo $(\beta=0,19)$. Y en adultos mayores encontraron que la apertura a la experiencia era un predictor de la habilidad tipo Pensamiento visuoespacial $(\beta=0,21)$.

\section{Responsabilidad}

\section{Función ejecutiva}

En el estudio de Williams, Suchy y Kraybill (2010), la responsabilidad presentó una correlación de 0,04 con la medida de funcionamiento ejecutivo. Esta fue la correlación más baja en comparación con los demás factores. También analizaron las correlaciones entre las facetas y la función ejecutiva. Las correlaciones fueron las siguientes: competencia $(0,22)$, sentido del deber $(0,04)$, esfuerzo por el logro $(-0,02)$, orden $(-0,10)$, autodisciplina $(-0,03)$, y deliberación $(0,10)$. 
DeYoung, Peterson y Higgins (2005) no encontraron ningún tipo de asociación $(0,01)$ entre el factor responsabilidad y el funcionamiento ejecutivo.

Las personas con altos niveles en la faceta de "orden" son metódicas, sistemáticas y disciplinadas (Chamorro-Premuzic y Furnham, 2003). La capacidad para definir un método organizado que pueda ser sistemáticamente ejecutado y la disciplina necesaria para cumplirlo son componentes cognitivos y comportamentales que están ligados al funcionamiento ejecutivo (Levin, Eisenberg y Benton, 1991). Por el contrario, las personas que son incapaces de diseñar una estrategia para conseguir un objetivo y tienen disicultades para ejecutarla de forma sistemática y organizada, podrían tener alteraciones en alguno de los componentes de la función ejecutiva (Cherkes-Julkowski, 2005).

Las personas con altos niveles de responsabilidad tienden a deliberar y no a actuar de forma reactiva. La deliberación se relaciona con la organización, planeación y regulación del comportamiento (DeYoung, 2010). La deliberación se contrapone a la impulsividad. Las personas impulsivas tienden a actuar rápidamente y sin que exista una programación previa. Los individuos impulsivos carecen de la capacidad para planear y prever consecuencias. Son incapaces de anticipar las consecuencias de sus actos (Cherkes-Julkowski, 2005).

Las personas con altos niveles en el rasgo tienen un elevado sentido del deber. El sentido del deber está estrechamente asociado con la autodisciplina, la cual se refiere a la capacidad que tiene la persona para regular su propio comportamiento y autodeterminar sus objetivos, planes y procedimientos. Rabin, Fogel y Nutter-Upham (2011) analizaron los componentes neuropsicológicos de la procrastinación, que es el extremo opuesto de la faceta del sentio del deber.

Rabin, Fogel y Nutter-Upham (2011) consideran que la procrastinación involucra fallos en la autoregulación y la volición, aspectos controlados por las funciones ejecutivas, ya que los individuos con bajos puntajes en la faceta del sentido del deber tienden a dilatar o a posponer sus responsabilidades, llegando incluso a no completarlas. Los resultados demostraron que las personas con alto niveles de procrastinación tenían deficiencias en los procesos de iniciación, planificación, organización, regulación, memoria de trabajo, inhibición y monitorización.

\section{Memoria}

Merema, Speelman, Foster y Kaczmarek (2013) estudiaron las relaciones entre la responsabilidad y la memoria verbal y visual en una muestra de 177 adultos mayores con edades entre los 66 y los 90 años. El valor de la correlación entre la responsabilidad y la memoria visual fue de 0,117 , mientras que el valor de la correlación entre el factor y la memoria verbal fue de 0,047. En esta investigación también se analizó la relación entre el nivel de responsabilidad y el olvido autopercibido o los problemas de memoria autoreportados. La correlación para estas dos variables fue estadísticamente significativa $(\mathrm{p}<0,001)$ y tuvo un valor de 0,336 .

\section{Inteligencia}

Los resultados de los análisis correlacionales, en la investigación de Bartels et al. (2012), presentada en el apartado "Memoria" de "Neuroticismo", mostraron una correlación de 0,02 entre la responsabilidad y el ci total, una correlación de 0,01 entre la responsabilidad y el CI manipulativo, y una correlación de 0,02 entre la responsabilidad y el CI verbal. La correlación entre la responsabilidad y la medida combinada de inteligencia fue de $0,15(\mathrm{p}<0,01)$ en los estudiantes de grado medio; y la correlación entre la responsabilidad y la medida combinada de inteligencia fue de $0,12(p<0,05)$ para los estudiantes de grado alto, en el estudio de Lounsbury, Welsh, Gibson y Sundstrom (2005), presentada en el apartado "Memoria" de "Neuroticismo".

\section{Conclusiones}

Dado que es un área de estudio reciente y debido al tipo de revisión realizado, no es posible realizar una conclusión precisa. Es decir, no es posible establecer, en el estado del arte actual, cómo se correlacionan los factores y facetas de la personalidad con la función ejecutiva, la inteligencia y la memoria. Sí hay evidencia, la mayor parte de ella aquí presentada y discutida, sobre la existencia de correlatos neuropsicológicos y cognitivos en la función ejecutiva, la memoria y la inteligencia para cada uno de los cinco grandes factores de la personalidad. Lo que no puede establecerse aún, ya que se requeriría un número mayor de estudios y un metaanálisis cuantitativo, es cuál es la correlación real entre cada una de estas variables.

En términos generales puede afirmarse que existe un perfil neuropsicológico diferente para cada uno de los factores. Es decir, los procesos ejecutivos, atencionales, mnémicos e intelectuales se configuran de forma diferente según cada factor de personalidad. 
Por ejemplo, tal como se presentó, parece ser que las personas con altos niveles de neuroticismo tienen dificultades para la inhibición de respuestas, mientras que las personas con altos niveles de amabilidad tienen una buena capacidad para controlar los impulsos.

Con regularidad a lo largo de los diferentes estudios, las correlaciones entre los factores o facetas y las variables neuropsicológicas tenían coeficientes de correlación bajos, casi siempre entre 0,1 y 0,3 . En algunos casos, inclusive, no existía correlación. Estos valores no deben preocupar. Pese a la diversidad en las metodologías y técnicas utilizadas para evaluar las variables neuropsicológicas, se encontró como constante la existencia de una correlación. Este dato es, por sí mismo, valioso. Este hallazgo empírico asociado con la efectiva correlación entre la personalidad y el funcionamiento neuropsicológico está validado por las suposiciones teóricas sobre la relación entre el funcionamiento cerebral (bioquímico, neurobiológico, psicobiológico y neuropsicológico) y la forma como se organiza la personalidad.

\section{Referencias}

Ahadi, S. A. y Rothbart, M. K. (1994). En C. F. Halverson, G. A. Kohnstamm y R. P. Martin (Eds.), The Developing Structure of Temperament and Personality from Infancy to Adulthood (pp. 189-207). Hillsdale, NJ, USA: Laurence Erlbaum.

Alperson, B. D. (1968). Color association values and response interference on variants of the Stroop test. Acta Psychologica, 26(3), 286-295.

Ashton, M. C., Lee, K., Vernon, P. A. y Jang, K. L. (2000). Fluid intelligence, crystallized intelligence, and the openness/intellect factor. Journal of Research in Personality, 34(2), 198-207. doi: http://dx.doi.org/10.1006/ jrpe.1999.2276

Baddeley, A. D. (1986). Working memory. New York: Oxford University Press.

Bagby, R. M. y Parker, D. A. (2001). Relation of rumination and distraction with neuroticism and extraversion in a sample of patients with major depression. Cognitive Therapy and Research, 25(1), 91-102. doi: http://dx. doi.org/10.1023/A:1026430900363

Baker, J. y Bichsel, J. (2006). Personality predictors of intelligence: Differences between young and cognitively healthy older adults. Personality and Individual Differences, 41(5), 861-871. doi: http://dx.doi.org/10.1016/ j.paid.2006.02.017
Bartels, M., van Weegen, F. I., van Beijsterveldt, C. E. M., Carlier, M., Tinca J.C. Polderman, T. J. C., Hoekstra, R. A. y Boomsma, D. I. (2012). The five factor model of personality and intelligence: A twin study on the relationship between the two constructs. Personality and Individual Differences, 53(4), 368-373. doi: http:// dx.doi.org/10.1016/j.paid.2012.02.007

Belojevic, G., Jakovljevic, B. y Slepcevic, V. (2003). Noise and mental performance: Personality attributes and noise sensitivity. Noise and Health, 6(21), 77-89.

Berggren, N., Koster, E. W. H. y Derakshan, N. (2012). The effect of cognitive load in emotional attention and trait anxiety: An eye-movement study. Journal of Cognitive Psychology, 24(1), 79-91. doi: http://dx.doi.org/10.108 0/20445911.2011.618450

Bermúdez, J., Pérez, A. M. y Padilla, M. (1988). Extraversion and task properties as determinants of incidental recall. European Journal of Personality, 2(1), 57-65. doi: http://dx.doi.org/10.1002/per.2410020105

Bettencourt, B. A., Talley, A., Benjamin, A. J. y Valentine, J. (2006). Personality and Aggressive Behavior Under Provoking and Neutral Conditions: A Meta-Analytic Review. Psychological Bulletin, 132(5), 751-777. doi: http://dx.doi.org/10.1037/0033-2909.132.5.751

Bishop, S. J. (2007). Neurocognitive mechanisms of anxiety: an integrative account. Trends in Cognitive Sciences, 11, 307-316. doi: http://dx.doi.org/10.1016/j.tics.2007. 05.008

Bishop, S. J. (2009). Trait anxiety and impoverished prefrontal control of attention. Nature Neuroscience 12, 92-98. doi: http://dx.doi.org/10.1038/nn.2242

Bono, J. E. y Vey, M. A. (2007). Personality and emotional performance: extraversion, neuroticism, and self-monitoring. Journal of Occupational and Health Psychology, 12(2), 177-192. doi: http://dx.doi.org/10.1037/ 1076-8998.12.2.177

Botvinick, M. M., Braver, T. S., Barch, D. M., Carter, C. S. y Cohen, J. D. (2001). Conflict monitoring and cognitive control. Psychological Review, 108(3), 624-652. doi: http://dx.doi.org/10.1037/0033-295X.108.3.624

Boyle, L. L., Lyness, J. M., Duberstein, P. R., Karuza, J., King, D. A., Messing, S., y Tu, X. (2010). Trait Neuroticism, Depression, and Cognitive Function in Older Primary Care Patients. American Journal of Geriatric Psychiatry, 18(4), 305-312. doi: http://dx.doi.org/10.1097/ JGP.0b013e3181c2941b

Brebner, J. (1985). Personality theory and movement. En B. D. Kirkcaldy (Ed.), Individual differences in movement (pp. 27-41). Lancaster, England: мтP Press Limited. doi: http://dx.doi.org/10.1007/978-94-009-4912-6_2 
Brebner, J. M. (1980). Reaction time in personality theory. En A. T. Welford (Ed.), Reaction times (pp. 309-320). London, England: Academic Press.

Bredemeier, K., Berenbaum, H., Most, S. B. y Simons, D. J. (2011). Links between neuroticism, emotional distress, and disengaging attention: Evidence from a single-target RSvP task. Cognition and Emotion, 25(8), 1510-1519. doi: http://dx.doi.org/10.1080/02699931. 2010.549460

Buela Casal, G., Caballo, V. E., García Cueto, E. y Flores Cubos, P. (1990). Attention and reaction time differences in introversion-extraversion. Personality and Individual Differences, 11(2), 195-197. doi: http://dx.doi. org/10.1016/0191-8869(90)90015-J

Campbell, A. M., Davalos, D. B., McCabe, D. P. y Troup, L. J. (2011). Executive functions and extraversion. Personality and Individual Differences, 51(6), 720-725. doi: 10.1016/j.paid.2011.06.018

Campbell, L., Simpson, J., Stewart, M. y Manning, J. (2003). Putting Personality in Social Context: Extraversion, Emergent Leadership, and the Availability of Rewards. Personality and Social Psychology Bulletin, 29(12), 1547-1559. doi: 10.1177/0146167203256920

Carver, C. S. y White, T. (1994). Behavioral inhibition, behavioral activation, and affective responses to impending reward and punishment: The BIS/BAs scales. Journal of Personality \& Social Psychology, 67(2), 319-333. doi: http://dx.doi.org/10.1037/0022-3514.67.2.319

Chamorro-Premuzic, T. y Furnham, A. (2003). Personality predicts academic performance: Evidence from two longitudinal university samples, Journal of Research in Personality, 37(4), 319-338. doi: 10.1016/S0092-6566 (02)00578-0

Cherkes-Julkowski, M. (2005). The dysfunctionality of executive function. Kearney, NE, USA: Morris Publishing.

Colom, R. y Quiroga, M. A. (2009). Neuroticism, intelligence, and intra-individual variability in elementary cognitive tasks: Testing the mental noise hypothesis. Psicothema, 21(3), 403-408.

Costa, P. T. y McCrae, R. (1992). The NEO PI-R professional manual. Odessa, FL: Psychological Assesment Resources.

Damasio, A. R. (1996). Descartes' error. Emotion, reason, and the human brain. New York: Putnam.

Davis, R. N. y Nolen-Hoeksema, S. (2000). Cognitive Inflexibility Among Ruminators and Nonruminators. Cognitive Therapy and Research, 24(6), 699-711. doi: http://dx.doi.org/10.1023/A:1005591412406
Denburg, N. L., Weller, J. A., Yamada, T. H., Shivapour, D. M., Kaup, A. R., LaLoggia, A., Cole, C. A., Tranel, D. y Bechara, A. (2009). Poor decision making among older adults is related to elevated levels of neuroticism. Annals of Behavioral Medicine, 37(2), 164-172. doi: 10.1007/s12160-009-9094-7

Depue, R. A. e Iacono, W. G. (1989). Neurobehavioral aspects of affective disorders. Annual Review of Psychology, 40, 457-492. doi: http://dx.doi.org/10.1146/ annurev.ps.40.020189.002325

Derakshan, N. y Eysenck, M. W. (2009). Anxiety, processing efficiency, and cognitive performance: new developments from attentional control theory. European Psychologist, 14(2), 168-176. doi: 10.1027/10169040.14.2.168

Derakshan, N., Smyth, S. y Eysenck, M. W. (2009). Effects of state anxiety on performance using a task-switching paradigm: an investigation of attentional control theory. Psychonomic Bulletin and Review, 16(6), 1112 1117. doi: 10.3758/PBR.16.6.1112.

DeYoung, C. G. (2010). Impulsivity as a personality trait. En K. D. Vohs y R. F. Baumeister (Eds.), Handbook of self-regulation: Research, theory, and applications (2. ed., pp. 485-504). New York, NY: Guilford Press.

DeYoung, C. G., Peterson, J. B., y Higgins, D. M. (2005). Sources of openness/intellect: Cognitive and neuropsychological correlates of the fifth factor of personality. Journal of Personality, 73(4), 825-858. doi: http://dx.doi.org/10.1111/j.1467-6494.2005.00330.x

Dreisbach, G. y Goschke, T. (2004). How positive affect modulates cognitive control: Reduced perseveration at the cost of increased distractibility. Journal of Experimental Psychology: Learning, Memory and Cognition, 30(2), 343-353. doi: http://dx.doi.org/10.1037/02787393.30 .2 .343

Duncker, K. (1945). On Problem-solving. Psychological Monographs, 58(5). i-113. doi: http://dx.doi.org/10.1037/ h0093599

Eisenberg, N., Fabes, F. A., Murphy, B., Karbon, M., Smith, M. y Maszk, P. (1996). The relations of children's dispositional empathy-related responding to their emotionality, regulation, and social functioning. Developmental Psychology, 32(2), 195-209.

Eysenck, H. J. (1967). The biological bases of personality. Baltimore: University Park Press.

Eysenck, M. W. y Derakshan, N. (2011). New perspectives in attentional control theory. Personality and Individual Differences, 50(7), 955-960. doi: 10.1016/j.paid. 2010.08.019 
Eysenck, M. W., Derakshan, N., Santos, R. y Calvo, M. G. (2007). Anxiety and cognitive performance: attentional control theory. Emotion, 7(2), 336-353. doi: http:// dx.doi.org/10.1037/1528-3542.7.2.336

Eysenck, M. W., Payne, S. y Derakshan, N. (2005). Trait anxiety, visuospatial processing, and working memory. Cognition and Emotion, 19(8), 1214-1228. doi: http://dx.doi.org/10.1080/02699930500260245

Feldman Barrett, L. (1997). The relationship among momentary emotion experiences, personality descriptions, and retrospective ratings of emotion. Personality and Social Psychology Bulletin, 23(10), 1100-1110. doi: http://dx.doi.org/10.1177/01461672972310010

Flehmig, H. C., Steinborn, M., Langner, R. y Westhoff, K. (2007). Neuroticism and the mental noise hypothesis: Relationships to lapses of attention and slips of action in everyday life. Psychology Science, 49(4), 343-360.

Flory, J. D., Harvey, P. D., Mitropoulo, V., New, A. S., Silverman, J. M., Siever, L. S. y Manuck, S. (2006). Dispositional impulsivity in normal and abnormal samples. Journal of Psychiatric Research, 40(5), 438-447. doi: http://dx.doi.org/10.1016/j.jpsychires.2006.01.008

Frost, P., Sparrow, S. y Barry, J. (2006). Personality Characteristics Associated with Susceptibility to False Memories. The American Journal of Psychology, 119(2), 193-204. doi: http://dx.doi.org/10.2307/20445334

Furnham, A., Forde, L., y Cotter, T. (1998). Personality and intelligence. Personality and Individual Differences, 24(2), 187-192.

Gabrys, J. B., Schumph, D. y Utendale, K. A. (1987). Short-term memory for two meaningful stories and self-report on the Adult Eysenck Personality Questionnaire. Psychological Reports, 61(1), 51-59. doi: 10.2466/pr0.1987.61.1.51

García, D. y Muñoz, P. (2000). Funciones ejecutivas y rendimiento escolar en educación primaria. Un estudio exploratorio. Revista Complutense de Educación, 11(1), 39-56.

García-Andrés, E., Huertas-Martínez, J. A., Ardura, A. y Fernández-Alcaraz, C. (2010). Emotional regulation and executive function profiles of functioning related to the social development of children. Procedia - Social and Behavioral Sciences, 5, 2077-2081. doi: 10.1016/j. sbspro.2010.07.416

Goel, V. y Grafman, J. (1995). Are the frontal lobes implicated in 'planning' functions? Interpreting data from the Tower of Hanoi. Neuropsychologia, 33(5), 623-642. doi: 10.1016/0028-3932(95)90866-P
Goldstein, D. G. y Gigerenzer, G. (2002). Models of ecological rationality: The recognition heuristic. Psychological Review, 109(1), 75-90. doi: 10.1037//0033-295X.109.1.75

Gray, J. (2009). The association between empathy, the Big 5 Dimensions of personality and prosocial behaviour: What causes individuals to act prosocially? Word Journal of The International Linguistic Associatio (Tesis de pregrado, Universidad de Edinburgo).

Gray, J. A. (1982). The neuropsychology of anxiety: An enquiry into the functions of the septohippocampal system. Oxford, England: Oxford University Press.

Gray, J. A. (1987). Thepsychologyoffearandstress. Cambridge, England: Cambridge University Press.

Gray, J. A. (1990). Brain systems that mediate both emotion and cognition. Cognition and Emotion, 4, 269-288. doi: 10.1080/02699939008410799

Gray, J. R. y Braver, T. S. (2002). Personality predicts working-memory-related activation in the caudal anterior cingulate cortex. Cognitive, Affective \& Behavioral Neuroscience, 2(1), 64-75. doi: http://dx.doi.org/10.3758/ CABN.2.1.64

Gupta, B. S. y Kumar, J. (1990). Effects of extraversion and elaboration of encoding on immediate free recall and recognition performance. Psychological Studies, 35(1), 46-51.

Harley, T. A. y Matthews, G. (1992). Interactive effects of extraversion, arousal and time of day on semantic priming: Are they pre-lexical or post-lexical? Personality and Individual Differences, 13(9), 1021-1029. doi: 10.1016/0191-8869(92)90135-C

Helode, R. D. (1982). Cognitive and non cognitive correlate of the Stroop interference effect. Journal of Psychological Researches, 26(3), 142-145.

Hervas, G. y Vazquez, C. (2011). What Else Do You Feel When You Feel Sad? Emotional Overproduction, Neuroticism and Rumination. Emotion, 11(4), 881-895. doi: $10.1037 / \mathrm{a} 0021770$

Hilbig, B. E. (2008). Individual differences in fast-and-frugal decision making: Neuroticism and the recognition heuristic. Journal of Research in Personality, 42(5), 1641-1645. doi: 10.1016/j.jrp.2008.07.001

Holland, D. C., Dollinger, S. J., Holland, C. J. y MacDonald, D. A. (1995). The relationship between psychometric intelligence and the five-factor model of personality in a rehabilitation sample. Journal of Clinical Psychology, 51(1), 79-88. doi: http://dx.doi.org/10.1002/1097-4679 (199501)51:1<79::AID-JCLP2270510113>3.0.CO;2-P 
Horn, J. L. (1985). Remodeling old models of intelligence. En B. B. Wolman (Ed.), Handbook of intelligence (pp. 267-300). New York: Wiley.

Howarth, E. y Eysenck, H. J. (1968). Extraversion, arousal and paired-associates recall. Journal of Experimental Research in Personality, 3(2), 114-116.

Ingram, R. E. (1990). Self-focused attention in clinical disorders: Review and a conceptual model. Psychological Bulletin, 107(2), 156-176. doi: http://dx.doi.org/ 10.1037/0033-2909.107.2.156

Isen, A. M. (1984). The influence of positive affect on decision making and cognitive organization. Advances in Consumer Research, 11, 534-537.

Isen, A. M. y Daubman, K. A. (1984). The influence of affect on categorization. Journal of Personality and Social Psychology, 47(6), 1206-1217. doi: http://dx.doi. org/10.1037/0022-3514.47.6.1206

Isen, A. M., Daubman, K. A. y Nowicki, G. P. (1987). Positive affect facilitates creative problem solving. Journal of Personality and Social Psychology, 52(6), 1122-1131. doi: http://dx.doi.org/10.1037/0022-3514.52.6.1122

Isen, A. M. y Means, B. (1983). The influence of positive affect on decision-making strategy. Social Cognition, 2(1), 18-31. doi: 10.1521/soco.1983.2.1.18

Isen, A. M., Niedenthal, P. M. y Cantor, N. (1992). An influence of positive affect on social categorization. Motivation and Emotion, 16(1), 65-78. doi: 10.1007/ BF00996487

Jelicic, M., Bosma, H., Ponds, R. W. H. M., Van Boxtel, M. P. J., Houx, P. J. y Jolles, J. (2003). Neuroticism does not affect cognitive functioning in later life. Experimental Aging Research, 29(1), 73-78. doi: http://dx.doi. org/10.1080/03610730303704

Jensen-Campbell, L. A., Rosseli, M., Workman, K. A., Santisi, M., Rios, J. D. y Bojan, D. (2002). Agreeableness, conscientiousness and effortful control processes. Journal of Research in Personality, 36(5), 476-489. doi: http://dx.doi.org/10.1016/S0092-6566(02)00004-1

Jensen, A. R. (1992). The importance of intraindividual variability in reaction time. Personality and Individual Differences, 13(8), 869-882. doi: http://dx.doi. org/10.1016/0191-8869(92)90004-9

Jensen, A. R. (2007). Clocking the mind. The Netherlands: Elsevier.

Jorm, A. F., Mackinnon , A. J., Christensen, H., Henderson, S., Scott, R. y Korten, A. (1993). Cognitive functioning and neuroticism in an elderly community sample. Personality and Individual Differences, 15(6), 721-723. doi: http://dx.doi.org/10.1016/0191-8869(93)90013-S
Joseph, R. M. y Tager-Flusberg, H. (2004). The relationship of theory of mind and executive functions to symptom type and severity in children with autism. Development and Psychopatholgy, 16(1), 137-155. doi: http:// dx.doi.org/10.1017/s095457940404444x

Koelega, H. S. (1992). Extraversion and vigilance performance: 30 years of inconsistencies. Psychological Bulletin, 112(2), 239-258. doi: http://dx.doi.org/10.1037/00332909.112.2.239

Koster, E. H., Raedt, R. D., Goeleven, E., Franck, E. y Crombez, G. (2005). Mood-congruent attentional bias in dysphoria: Maintained attention to and delayed engagement from negative information. Emotion, 5(4), 446455. doi: http://dx.doi.org/10.1037/1528-3542.5.4.446

Levin, H. S., Eisenberg, H. M. y Benton, A. L. (1991). Frontal lobe function and dysfunction. New York: Oxford University Press.

Lieberman, M. D. (2000). Introversion and working memory: central executive differences. Personality and Individual Dfferences, 28(3), 479-486. doi: http://dx.doi. org/10.1016/S0191-8869(99)00113-0

Lieberman, M. D. y Rosenthal, R. (2001). Why introverts can't always tell who likes them: Multitasking and nonverbal decoding. Journal of Personality \& Social Psychology, 80(2), 294-310. doi: http://dx.doi.org/ 10.1037/0022-3514.80.2.294

Lounsbury, J. W., Welsh, D. P., Gibson, L. W. y Sundstrom, E. (2005). Broad and narrow personality traits in relation to cognitive ability in adolescents. Personality and Individual Differences, 38(5),1009-1019. doi: http:// dx.doi.org/10.1016/j.paid.2004.06.022

Luu, P., Collins, P. y Tucker, D. M. (2000). Mood, personality, and self-monitoring: Negative affect and emotionality in relation to frontal lobe mechanisms of error monitoring. Journal of Experimental Psychology: General, 129(1), 43-60. doi: http://dx.doi.org/10.1037/00963445.129 .1 .43

Lyubomirsky, S. y Nolen-Hoeksema, S. (1995). Effects of self-focused rumination on negative thinking and interpersonal problem solving. Journal of Personality and Social Psychology, 69(1), 176-190. doi: http://dx. doi.org/10.1037/0022-3514.69.1.176

Lyubomirsky, S., Caldwell, N. D. y Nolen-Hoeksema, S. (1998). Effects of ruminative and distracting responses to depressed mood on the retrieval of autobiographical memories. Journal of Personality and Social Psychology, 75(1), 166-177. doi: http://dx.doi.org/10.1037/00223514.75.1.166 
Lyubomirsky, S., Tucker, K. L., Caldwell, N. D. y Berg, K. (1999). Why ruminators are poor problem solvers: Clues from the phenomenology of dysphoric rumination. Journal of Personality and Social Psychology, 77(5), 1041-1060. doi: http://dx.doi.org/10.1037/00223514. 77.5 .1041

Matthews, G., Davies, D. R. y Lees, J. L. (1990). Arousal, extraversion, and individual differences in resource availability. Journal of Personality and Social Psychology, 50(1), 150-168. doi: http://dx.doi.org/10.1037/00223514.59 .1 .150

McCrae, R. R. (1993-1994). Openness to Experience as a basic dimension of personality. Imagination, Cognition and Personality, 13(1), 39-55. doi: http://dx.doi. org/10.2190/H8H6-QYKR-KEU8-GAQ0

Merema, M. R., Speelman, C. P., Foster, J. K. y Kaczmarek, E. A. (2013). Neuroticism (Not Depressive Symptoms) Predicts Memory Complaints in Some Community-Dwelling Older Adults. The American Journal of Geriatric Psychiatry, 21(8), 729-736. doi: http://dx.doi. org/10.1016/j.jagp.2013.01.059

Muris, P. E., Bos, A. E., Mayer, B. N., Verkade, R., Thewissen, V. y Dell'Avvento, V. (2009). Relations among behavioral inhibition, Big Five personality factors, and anxiety disorder symptoms in non-clinical children. Personality and Individual Differences, 46(4), 525-529. doi: http://dx.doi.org/10.1016/j.paid.2008.12.003

Muris, P. E., De Jong, P. J. y Engelen, S. (2004). Relationships between neuroticism, attentional control, and anxiety disorders symptoms in non-clinical children. Personality and Individual Differences, 37(4), 789-797. doi: http://dx.doi.org/10.1016/j.paid.2003.10.007

Muris, P., Roelofs, J., Rassin, E., Franken, I. y Mayer, B. (2005). Mediating effects of rumination and worry on the links between neuroticism, anxiety and depression. Personality and Individual Differences, 39(6), 1105-1111. doi: http://dx.doi.org/10.1016/j.paid.2005. 04.005

Nolen-Hoeksema, S. (2000). The role of rumination in depressive disorders and mixed anxiety/depressive symptoms. Journal of Abnormal Psychology, 109(3), 504-511. doi: http://dx.doi.org/10.1037/0021-843X.109.3.504

O’Brien, T. B. y DeLongis, A. (1996). The interactional context of problem-, emotion-, and relationship- focused coping: The role of the Big Five personality factors. Journal of Personality, 64(4), 775-813. doi: http://dx. doi.org/10.1111/j.1467-6494.1996.tb00944.x
Ode, S. y Robinson, M. D. (2007). Agreeableness and the self-regulation of negative affect: Findings involving the neuroticism/somatic distress relationship. Personality and Individual Differences, 43(8), 2137-2148. doi: http://dx.doi.org/10.1016/j.paid.2007.06.035

Pearson, P. R. (1993). Cognitive functioning and neuroticism in elderly psychiatric patients. Personality and Individual Differences, 14(1), 265-266. doi: http://dx.doi. org/10.1016/0191-8869(93)90201-D

Portella, M. J., Goodwin, G. M., Flint, J., Cowen, P. y Harmer, C. J. (2004). Neuroticism as a psychological vulnerability factor for mood disorders. Journal of Psychopharmacology, 18(3), A12.

Posner, P. I. y Petersen, S. E. (1990). The attention system of the human brain. Annual Review of Neuroscience, 13, 25-42. doi: http://dx.doi.org/10.1146/annurev.ne. 13.030190 .000325

Puskar, A. (2011). Investigation of the relationship between the cognitive and affective components of empathy and frontal lobe functioning in college students (Doctoral Dissertation, Indiana University of Pennsylvania, Pennsylvania).

Rabin, L. A., Fogel, J. y Nutter-Upham, K. E. (2011). Academic procrastination in college students: The role of self-reported executive function. Journal of Clinical and Experimental Neuropsychology, 33(3), 344-357. doi: http://dx.doi.org/10.1080/13803395.2010.518597

Ramchandran, K. (2011). The neuropsychological correlates of leadership effectiveness. Theses and Dissertations. Iowa: University of Iowa.

Rameson, L. T. y Lieberman, M. D. (2009). Empathy: A Social Cognitive Neuroscience Approach. Social and Personality Psychology Compass, 3(1), 94-110. doi: http:// dx.doi.org/10.1111/j.1751-9004.2008.00154.x

Rasmussen, C., Wyper, K. y Talwar, V. (2009). The relation between theory of mind and executive functions in children with fetal alcohol spectrum disorders. Canadian Journal of Clinical Pharmacology, 16(2), e370-380.

Reeve, W. V. y Schnadler, S. L. (2001). Frontal lobe functioning in adolescents with attention deficit hyperactivity disorder. Adolescence, 36(144), 749-765.

Roberts, J. E., Gilboa, E. y Gotlib, I. H. (1998). Ruminative response style and vulnerability to episodes of dysphoria: Gender, neuroticism, and episode duration. Cognitive Therapy and Research, 22(4), 401-423. doi: http:// dx.doi.org/10.1023/A:1018713313894 
Roberts, M. J. (2002). The relationship between extraversion and ability. Personality and Individual Differences, 32(3), 517-522. doi: http://dx.doi.org/10.1016/S01918869(01)00053-8

Robinson, M. D. y Tamir, M. (2005). Neuroticism as mental noise: a relation between neuroticism and reaction time standard deviations. Journal of Personality and Social Psychology, 89(1), 107-114. doi: http://dx.doi. org/10.1037/0022-3514.89.1.107

Rothbart, M. K. y Bates, J. E. (2006). Temperament. Handbook of child psychology. En N. Eisenberg \& W. Damon (Eds.), Social, emotional, and personality development (6th ed., Vol. 3, pp. 99-166). New York: Wiley.

Safer, M. A. y Keuler, D. J. (2002). Individual differences in misremembering pre-psychotherapy distress: Personality and memory distortion. Emotion, 2(2), 162-178. doi: http://dx.doi.org/10.1037/1528-3542.2.2.162

Safer, M. A., Levine, L. J. y Drapalski, A. L. (2002). Distortion in Memory for Emotions: The Contributions of Personality and Post-Event Knowledge. Personality and Social Psychology Bulletin, 28(11), 1495-1507. doi: http:// dx.doi.org/10.1177/014616702237577

Salthouse, T. A., Atkinson, T. M. y Berish, D. E. (2003). Executive functioning as a potential mediator of age-related cognitive decline in normal adults. Journal of Experimental Psychology: General, 132(4), 566-594. doi: http://dx.doi.org/10.1037/0096-3445.132.4.566

Sanford, L. C. y Fisk, J. E. (2009). How does the extraversion personality trait influence false recall with the DeeseRoediger-McDermott (DRM) paradigm? Journal of Research in Personality, 43(6), 972-977. doi: http://dx.doi. org/10.1016/j.jrp.2009.05.011

Schretlen, D. J., Van der Hulst, E. J., Pearlson, G. D. y Gordon, B. (2010). A neuropsychological study of personality: trait openness in relation to intelligence, fluency, and executive functioning. Journal of Clinical and Experimental Neuropsychology, 32(10), 1068-1073. doi: http://dx.doi.org/10.1080/13803391003689770

Schwartz, S. (1979). Differential effects of personality on access to various longterm memory codes. Journal of Research in Personality, 13(4), 396-403. doi: http://dx. doi.org/10.1016/0092-6566(79)90003-5

Smits, D. J. y Boeck, P. D. (2006). From BIs/Bas to the Big Five. European Journal of Personality, 20(4), 255-270. doi: http://dx.doi.org/10.1002/per.583

Stahl, J. y Gibbons, H. (2007). Dynamics of response-conflict monitoring and individual differences in response control and behavioral control: An electrophysiological investigation using a stop-signal paradigm. Clinical Neurophysiology, 118(3), 581-596. doi: http://dx.doi. org/10.1016/j.clinph.2006.10.023

Stahl, J. y Rammsayer, T. (2007). The point of no return in motor response: Extraversion-related differences. Psychology Science, 49(1), 58-67.

Stankov, L. (2009). Conservatism and cognitive ability. Intelligence, 37, 294-304. doi: http://dx.doi.org/10.1016/j. intell.2008.12.007

Stough, C., Brebner, J., Nettelbeck, T., Cooper, C. J., Bates, T. y Mangan, G. L. (1996). The relationship between intelligence, personality, and inspection time. British Journal of Psychology, 87(2), 255-268. doi: http://dx. doi.org/10.1111/j.2044-8295.1996.tb02589.x

Studer-Luethi, B., Jaeggi, S. M., Buschkuehl, M. y Perrig, W. J. (2012). Influence of neuroticism and conscientiousness on working memory training outcome. Personality and Individual Differences, 53(1), 44-49. doi: http:// dx.doi.org/10.1016/j.paid.2012.02.012

Tangney, J. P., Baumeister, R. F. y Boone, A. L. (2004). High self-control predicts good adjustment, less pathology, better grades, and interpersonal success. Journal of Personality, 72(2), 271-322. doi: http://dx.doi.org/10.1111/j.0022-3506.2004.00263.x

Teasdale, J. D. y Green, H. A. (2004). Ruminative self-focus and autobiographical memory. Personality and Individual Differences, 36(8), 1933-1943. doi: http://dx.doi. org/10.1016/j.paid.2003.08.022

Tobin, R., Graziano, W., Vanman, E. y Tassinary, L. (2000), Personality, emotional experience, and efforts to control emotions. Journal of Personality and Social Psychology, 79(4), 656-669. doi: http://dx.doi.org/10. 1037/0022-3514.79.4.656

Wainwright, M. A., Wright, M. J., Luciano, M., Geffen, G. M. y Martin, N. G. (2008). Genetic covariation among facets of openness to experience and general cognitive ability. Twin Research and Human Genetics, 11(3), 275286. doi: http://dx.doi.org/10.1375/twin.11.3.275

Wallace, J. E. y Newman, J. P. (1998). Neuroticism and the facilitation of automatic orienting of attention. Personality and Individual Differences, 24(2), 253-266. doi: http://dx.doi.org/10.1016/S0191-8869(97)00153-0

Watkins, E., y Moulds, M. L. (2005). Distinct modes of ruminative self-focus: Impact of abstract versus concrete rumination on problem solving in depression. Emotion 5(3), 319-328. doi: http://dx.doi.org/10.1037/15283542.5.3.319 